\title{
Exciton energy spectra in polyyne chains
}

\author{
Stella Kutrovskaya $\odot,{ }^{1,2,3}$ Sevak Demirchyan $\odot,{ }^{1,2}$ Anton Osipov $\odot,{ }^{3,4}$ Stepan Baryshev $\odot,{ }^{5}$ Anton Zasedatelev $\odot,{ }^{5,6}$ \\ Pavlos Lagoudakis $\odot, 5,6$ and Alexey Kavokin $\oplus^{1,2,7,8}$ \\ ${ }^{1}$ School of Science, Westlake University, Hangzhou 310024, Zhejiang Province, China \\ ${ }^{2}$ Institute of Natural Sciences, Westlake Institute for Advanced Study, Hangzhou 310024, Zhejiang Province, China \\ ${ }^{3}$ Department of Physics and Applied Mathematics, Stoletov Vladimir State University, Vladimir, Russia \\ ${ }^{4}$ ILIT RAS Branch of FSRC Crystallography and Photonics" RAS, Shatura 140700, Russia \\ ${ }^{5}$ Skolkovo Institute of Science and Technology, 121205 Moscow, Russia \\ ${ }^{6}$ Physics and Astronomy, University of Southampton, Highfield, Southampton, SO171BJ, United Kingdom \\ ${ }^{7}$ Russian Quantum Center, Skolkovo IC, Moscow 121205, Russia \\ ${ }^{8}$ NTI Center for Quantum Communications, National University of Science and Technology MISiS, Moscow 119049, Russia
}

(Received 4 August 2020; revised 31 October 2020; accepted 22 December 2020; published 22 January 2021)

\begin{abstract}
Recently, we have experimentally observed signatures of sharp exciton peaks in the photoluminescence spectra of bundles of monoatomic carbon chains stabilized by gold nanoparticles and deposited on a glass substrate. Here we estimate the characteristic energies of excitonic transitions in this complex quasi-one-dimensional nanosystem with use of the variational method. We show that the characteristic energy scale for the experimentally observed excitonic fine structure is governed by the interplay between the hopping energy in a Van der Waals quasicrystal formed by parallel carbon chains, neutral-charged exciton splitting, and positive-negative trion splitting. These three characteristic energies are an order of magnitude lower than the direct exciton binding energy.
\end{abstract}

DOI: 10.1103/PhysRevResearch.3.013071

\section{INTRODUCTION}

Being monoatomic chains of carbon atoms carbynes represent ultimate one-dimensional crystals. Carbynes are linear chains of $s p^{1}$-hybridized carbon atoms [1]. Their two known allotropes are polyyne, characterized by alternating single and triple electronic bonds between carbon atoms, and cumulene, characterized by double bonds between atoms. Theoretical and recent experimental studies indicate the semiconducting behavior for polyyne chains [2] and quasicrystals, while infinite cumulene chains are expected to be metallic $[3,4]$. The strain in finite-size chains of polyyne results in the increase of the direct band gap, so that experimentally achievable polyyne structures are expected to be emitting visible light in a wide spectral range that is dependent on the specific geometry of the structure $[5,6]$. This makes polyyne based nanostructures highly promising for the realization of light-emitting diodes and nanolasers. In order to predict the quantum efficiency of carbon-based optical nanoemitters one should learn more about excitons in linear carbon chains. The exciton binding energy in $s p^{1}$ carbon must be quite large due to the strong twodimensional quantum confinement as has been realized in the work [7]. Recently, we detected the strong excitonic features

Published by the American Physical Society under the terms of the Creative Commons Attribution 4.0 International license. Further distribution of this work must maintain attribution to the author(s) and the published article's title, journal citation, and DOI. in low-temperature photoluminescence spectra of polyyne bundles stabilized by gold nanoparticles and deposited on a glass substrate. The focus of our study was on elongated polyynic molecules, containing straight parts of even numbers of atoms (from 8 to 24) regularly separated with kinks. The synthesized $s p^{1}$-hybridization chains were packed in hexagon bundles characterized by the distance between neighboring parallel chains of $5.35 \AA$ [8]. The chains were hold together by the Van der Waals force [see the schematic in Fig. 1(a)]. They were grown by the laser ablation in liquid and deposited on a fused quartz substrate for the photoluminescence (PL) study. The chains were stabilized by gold anchors attached to their ends [8]. In low-temperature PL spectra, we have observed the characteristic triplet structure [Figs. 1(b) and 1(c)]. The triplet is invariably composed of a sharp intense peak accompanied by two broader satellites shifted by about 15 and $40 \mathrm{meV}$ [9] to the lower-energy side of the main peak, respectively. Very interestingly, the triplet structure is found to be nearly identical in carbon chains of different lengths. It is shifted as a whole with the band-gap variation as the length of the chain changed. We assign the observed sharp peaks to the optical transitions associated with neutral and charged excitons in polyyne bundles. Indeed, as any direct bandgap semiconductor, polyyne is expected to sustain excitons. Sharp resonances that emerge at low temperatures are clear signatures of the excitonic emission. Our time-resolved photoluminescence measurements confirm this assumption [9]. In Ref. [9] we attributed the main peak of every triple PL structure to the neutral exciton transition and two lower-energy satellites to charged exciton (trion) transitions, respectively. 
(a)

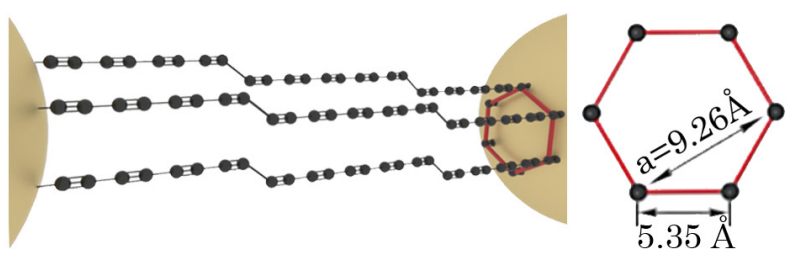

(b)

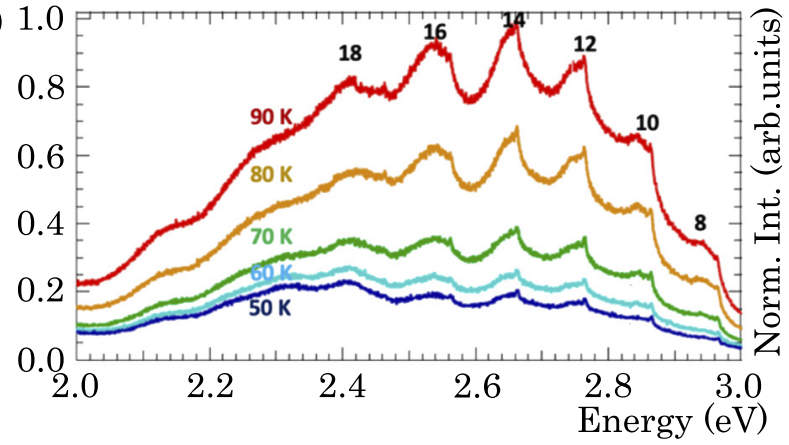

(c)

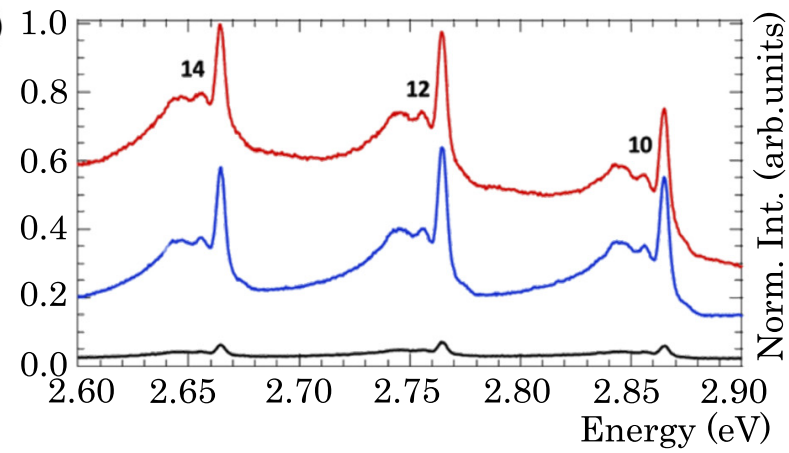

FIG. 1. The top panel (a) illustrates the concept of a stabilized bungle of polyyne chains endcapped by gold nanoparticles (shown as yellow spheres). It also shows the cross section of the structure that represents a hexagonal Van der Waals crystal. Panels (b) and (c) show the PL spectra of the deposited polyyne chains of different lengths (the number of atoms in the chain is indicated on the top of the corresponding spectral resonance). Panel (b) shows the PL spectra taken at temperatures between 90 and $50 \mathrm{~K}$, panel (c) shows the PL spectra taken at $4 \mathrm{~K}$ at different excitation wavelengths. Red, blue, and black curves correspond to the excitation wavelengths of 390,380 , and $370 \mathrm{~nm}$, respectively. The spectra are reproduced from Ref. [9].

The main argument supporting this interpretation was the detected dipole polarization of about a half of carbon-metal nanocomplexes that was revealed by their alignment in the presence of the external electric field [8].

To gain better understanding of the observed resonances, one needs to compare the anticipated exciton and trion energies in the system, estimate the thermal hopping energy and the splitting between positively and negatively charged trions. Here we attempt analyzing the energy spectra of excitons in carbyne-based nanosystems with use of the variational method. We rely on the effective mass approximation. Using this formalism we were able to shed light on the interplay between several characteristic energies that may be responsible for the fine structures observed experimentally, namely, the positive-negative trion energy splitting, the neutral-charged exciton energy splittings, and the hopping energy that splits spatially direct and indirect exciton states.

\section{EXCITON MODELING}

We recall that a Wannier-Mott exciton is a neutral quasiparticle [10], whose optical features strongly depend on the dimensionality of a semiconductor crystal [11]. Much attention has been focused on studies of excitons in strongly confined quantum systems $[12,13]$. Stronger confinements typically result in larger binding energies of excitons [14]. In addition to the exciton features, similar spectral resonances corresponding to negatively $\left(X^{-}\right)$and positively $\left(X^{+}\right)$charged exciton complexes (trions) consisting of two electrons and one hole and two holes and an electron, respectively, have been studies in doped semiconductor structures $[15,16]$. From the theoretical point of view, the excitonic problem is usually treated in the framework of the effective mass approximation [17]. Resolving the Schröedinger equation for the relative motion of electron and hole [18], one finds the fine structure of excitonic transition that varies from hydrogen-like, in bulk crystals [10], to 3D-quantum box spectra in small nanocrystals [13]. More challenging is the description of trion states, where a variety of many-body effects may come into play $[19,20]$. In the present study, we rely on the simplest quasianalytical approach, which seems to be the best adapted at the stage where very little is known about the electronic and optical properties of the relatively complex hybrid nanostructure under study. We extend the approaches developed in Refs. [18,21] for the variational calculations of exciton states and Ref. [22] for the analysis of trion states. We assume that electron and hole localization radii in the plane normal to the axis of the cylinder are given, respectively, by the parameters $R_{e}$ and $R_{h}$ that are much smaller than the exciton Bohr radius. In this case, the electron and hole confinement problem in the normal to the cylinder plane can be decoupled from the Schröedinger equation for the electron-hole relative motion along the axis of the cylinder. The finite size of the cylinder cross section matters as it is responsible for a finite value of the binding energy of a quasi-one-dimensional exciton [23]. We recall that in a purely one-dimensional limit the energy of a ground state of the Coulomb potential tends to minus infinity [24]. We shall work with the exciton trial wave function taken in form:

$$
X\left(\vec{\rho}_{h}, \vec{\rho}_{e}, z\right)=\Xi_{h}\left(\vec{\rho}_{h}\right) \Xi_{e}\left(\vec{\rho}_{e}\right) \Psi(z) .
$$

Here $\Xi_{h(e)}$ are hole (electron) wave functions in normal to the axis of the cylinder plane and $\Psi(z)$ is the wave function of the electron-hole relative motion along the axis of the cylinder. We multiply the exciton Schröedinger equation by the conjugate of the envelopes of the electron and hole wave functions and integrate over the corresponding coordinates. This enables us to derive the Schröedinger equation for an electron-hole relative motion function:

$$
H \Psi(z)=E \Psi(z),
$$

that contains a smoothed Coulomb potential:

$$
H=-\frac{\hbar^{2}}{2 \mu} \nabla_{z}^{2}-\frac{e^{2}}{4 \pi \varepsilon \varepsilon_{0}} \iint \frac{\left|\Xi_{h}\left(\vec{\rho}_{h}\right)\right|^{2}\left|\Xi_{e}\left(\vec{\rho}_{e}\right)\right|^{2}}{\sqrt{z^{2}+\left(\vec{\rho}_{h}-\vec{\rho}_{e}\right)^{2}}} d \vec{\rho}_{h} d \vec{\rho}_{e},
$$


where $\mu=\frac{m_{e} m_{h}}{m_{e}+m_{h}}$ is a reduced mass. The constituents of the exciton wave function are normalized to unity:

$$
\int_{-\infty}^{\infty}|\Psi(z)|^{2} d z=\int_{0}^{\infty} \int_{0}^{2 \pi}\left|\Xi_{h, e}\left(\vec{\rho}_{h, e}\right)\right|^{2} \rho_{h, e} d \rho_{h, e} d \phi_{h, e}=1 .
$$

For hole and electron envelopes we assume:

$$
\Xi_{h, e}\left(\vec{\rho}_{h, e}\right)=\frac{1}{\sqrt{\pi} R_{h, e}} \exp \left(-\frac{\rho_{h, e}^{2}}{2 R_{h, e}^{2}}\right) .
$$

Within these approximations the Schröedinger equation for the wave function of electron-hole relative motion becomes

$$
\begin{aligned}
& {\left[-\frac{\hbar^{2}}{2 \mu} \frac{\partial^{2}}{\partial z^{2}}-\frac{e^{2}}{4 \pi^{3} \varepsilon_{1} \varepsilon_{0} R_{h}^{2} R_{e}^{2}}\right.} \\
& \left.\quad \times \iint \frac{\exp \left(-\frac{\rho_{h}^{2}}{2 R_{h}^{2}}\right) \exp \left(-\frac{\rho_{e}^{2}}{2 R_{e}^{2}}\right) d \vec{\rho}_{h} d \vec{\rho}_{e}}{\sqrt{z^{2}+\rho_{e}^{2}+\rho_{h}^{2}-2 \rho_{h} \rho_{e} \cos \left(\phi_{e}-\phi_{h}\right)}}\right] \Psi(z) \\
& =E \Psi(z),
\end{aligned}
$$

where $\varepsilon_{1}$ is a dielectric constant corresponding to the spatially direct exciton confined inside the carbon chain. We solve Eq. (2) for $\Psi(z)$ by the variational method. The exciton energy minimization is carried out using the hydrogen-like trial function with a single variational parameter $a$ that has a meaning of the inverse Bohr-radius: $\Psi(z)=\sqrt{a} e^{-a|z|}$. We find $a$ by minimizing the energy functional $J(a)=\int_{-\infty}^{\infty} \Psi^{*}(z) H \Psi(z) d z$ that yields:

$$
\begin{aligned}
\frac{\hbar^{2} a}{\mu}= & \frac{e^{2}}{4 \pi^{3} \varepsilon_{1} \varepsilon_{0} R_{h}^{2} R_{e}^{2}} \int_{-\infty}^{\infty}(1-2 a|z|) \exp (-2 a|z|) \\
& \times \iint \frac{\exp \left(-\frac{\rho_{h}^{2}}{R_{h}^{2}}\right) \exp \left(-\frac{\rho_{e}^{2}}{R_{e}^{2}}\right) d \vec{\rho}_{h} d \vec{\rho}_{e}}{\sqrt{z^{2}+\rho_{h}^{2}+\rho_{e}^{2}-2 \rho_{h} \rho_{e} \cos \left(\phi_{e}-\phi_{h}\right)}} d z .
\end{aligned}
$$

For a spatially indirect exciton formed by an electron and a hole confined in neighboring cylinders we obtain:

$$
\begin{aligned}
\frac{\hbar^{2} a}{\mu}= & \frac{e^{2}}{4 \pi^{3} \varepsilon_{2} \varepsilon_{0} R_{h}^{2} R_{e}^{2}} \int_{-\infty}^{\infty}(1-2 a|z|) \exp (-2 a|z|) \\
& \times \iint \frac{\exp \left(-\frac{\rho_{h}^{2}+l^{2}-2 \rho_{h} l \sin \phi_{h}}{R_{h}^{2}}\right) \exp \left(-\frac{\rho_{e}^{2}}{R_{e}^{2}}\right) d \vec{\rho}_{h} d \vec{\rho}_{e}}{\sqrt{z^{2}+\rho_{h}^{2}+\rho_{e}^{2}-2 \rho_{h} \rho_{e} \cos \left(\phi_{e}-\phi_{h}\right)}} d z,
\end{aligned}
$$

where $l$ is the distance between the neighboring polyyne chains.

Next, we estimate the binding energy of positively and negatively charged trions. We represent the trial functions for positively and negatively charged trions in the form:

$$
\begin{aligned}
X^{ \pm} & \left(\vec{r}_{h 1, e 1}, \vec{r}_{h 2, e 2}, \vec{r}_{e, h}\right) \\
= & \Xi_{h 1, e 1}\left(\vec{\rho}_{h 1, e 1}\right) \\
& \times \Xi_{h 2, e 2}\left(\vec{\rho}_{h 2, e 2}\right) \Xi_{e, h}\left(\vec{\rho}_{e, h}\right) \Psi^{ \pm}\left(z_{h 1, e 1}, z_{h 2, e 2}, z_{e, h}\right) .
\end{aligned}
$$

The terms composing the trion wave functions are also normalized to unity and the electron and hole envelopes are assumed to be Gaussian isotropic functions, as before. The
Schröedinger equations for $X^{+}$and $X^{-}$states can be written as:

$$
\begin{aligned}
& {\left[-\frac{\hbar^{2}}{2 m_{h, e}} \frac{\partial^{2}}{\partial z_{h 1, e 1}^{2}}-\frac{\hbar^{2}}{2 m_{h, e}} \frac{\partial^{2}}{\partial z_{h 2, e 2}^{2}}-\frac{\hbar^{2}}{2 m_{e, h}} \frac{\partial^{2}}{\partial z_{e, h}^{2}}\right.} \\
& \left.\quad+V\left(z_{h 1, e 1}, z_{h 2, e 2}\right)-V\left(z_{h 1, e 1}, z_{e, h}\right)-V\left(z_{h 2, e 2}, z_{e, h}\right)\right] \\
& \quad \times \Psi^{ \pm}\left(z_{h 1, e 1}, z_{h 2, e 2}, z_{e, h}\right)=E^{ \pm} \Psi^{ \pm}\left(z_{h 1, e 1}, z_{h 2, e 2}, z_{e, h}\right),
\end{aligned}
$$

where

$$
\begin{aligned}
V\left(z_{\alpha}, z_{\beta}\right)= & \frac{e^{2}}{4 \pi^{3} \varepsilon_{1} \varepsilon_{0} R_{\alpha}^{2} R_{\beta}^{2}} \\
& \times \iint \frac{\exp \left(-\frac{\rho_{\alpha}^{2}}{R_{\alpha}^{2}}\right) \exp \left(-\frac{\rho_{\beta}^{2}}{R_{\beta}^{2}}\right) d \vec{\rho}_{\beta} d \vec{\rho}_{\alpha}}{\sqrt{\left(z_{\alpha}-z_{\beta}\right)^{2}+\left(\vec{\rho}_{\alpha}-\vec{\rho}_{\beta}\right)^{2}}}
\end{aligned}
$$

is the effective one-dimensional interaction potential. We assume that $R_{h 1}=R_{h 2}=R_{h}$ and $R_{e 1}=R_{e 2}=R_{e}$. It is convenient to introduce new coordinates of the relative motion. For $X^{+}$:

$$
z_{1}=z_{h 1}-z_{e}, z_{2}=z_{h 2}-z_{e}, Z=\frac{z_{h 1}+z_{h 2}+\frac{m_{e}}{m_{h}} z_{e}}{\frac{m_{e}}{m_{h}}+2} .
$$

The same is done for $X^{-}$replacing $h \rightarrow e$ and $e \rightarrow h$. Now we can separate the motion of a center of mass of a trion and the relative motion of particles and obtain the wave function of the relative motion of $X^{+}$and $X^{-}$trions:

$$
\begin{aligned}
& {\left[-\frac{\hbar^{2}}{2 \mu} \frac{\partial^{2}}{\partial z_{1}^{2}}-\frac{\hbar^{2}}{2 \mu} \frac{\partial^{2}}{\partial z_{2}^{2}}-\frac{\hbar^{2}}{m_{e, h}} \frac{\partial}{\partial z_{1}} \frac{\partial}{\partial z_{2}}\right.} \\
& \left.+U^{ \pm}\left(z_{1}, z_{2}\right)\right] \Psi^{ \pm}\left(z_{1}, z_{2}\right)=E^{ \pm} \Psi^{ \pm}\left(z_{1}, z_{2}\right) .
\end{aligned}
$$

Here the potentials of interaction in positively and negatively charged trions are introduced as $U^{+}\left(z_{1}, z_{2}\right)$ and $U^{-}\left(z_{1}, z_{2}\right)$, respectively.

In the following we shall focus on $X^{+}$and omit the superscript for simplicity. We shall solve the Schröedinger equation by the variational method assuming the trial function for trions in a singlet state having a form, where $c$ is a parameter describing the repulsion between similarly charged particles in trions:

$$
\Psi\left(z_{1}, z_{2}\right)=A e^{-a\left|z_{1}\right|} e^{-a\left|z_{2}\right|}\left(1+c\left|z_{1}-z_{2}\right|\right) .
$$

This wave function should be normalized to unity, which yields for $A$ :

$$
A=\sqrt{\frac{2 a^{4}}{2 a^{2}+3 c a+2 c^{2}}} .
$$

Now the energy functional to be minimized can be expressed as:

$$
\begin{aligned}
J(a, c)= & \frac{\hbar^{2}}{\mu} \frac{2 a^{4}+c a^{3}+2 c^{2} a^{2}}{2 a^{2}+3 c a+2 c^{2}}+\frac{\hbar^{2}}{m_{e}} \frac{c a^{3}-c^{2} a^{2}}{2 a^{2}+3 a c+2 c^{2}} \\
& +\frac{2 a^{4}}{2 a^{2}+3 c a+2 c^{2}} \int_{-\infty}^{\infty} \int_{-\infty}^{\infty} \exp \left(-2 a\left|z_{1}\right|\right) \\
& \times \exp \left(-2 a\left|z_{2}\right|\right) \\
& \times\left(1+c\left|z_{1}-z_{2}\right|\right)^{2} U\left(z_{1}, z_{2}\right) d z_{1} d z_{2} .
\end{aligned}
$$


In the case of $X^{-}$the functional is the same as before, with $m_{h}$ replacing $m_{e}$ in the second term. In order to prove the validity of a simple variational model formulated here, we have also performed the calculation accounting for the confinement of the exciton center-of-mass motion in a chain of a finite length as well as the calculations of trion states performed with a trial function having three independent variational parameters, see the supplemental material [25].

\section{RESULTS AND DISCUSSION}

Solving Eq. (7) with following fitting parameters $\varepsilon_{1}=6$, $R_{h}=1 \AA$, and $R_{e}=2.5 \AA$ and using the electron and hole effective masses predicted by the ab initio calculation [9]: $m_{e}=$ $0.078 m_{0}, m_{h}=0.09 m_{0}$, where $m_{0}$ is the free electron mass, we obtain for the spatially direct exciton $a=0.351 \mathrm{~nm}^{-1}$ and the binding energy $E_{X}=258,8 \mathrm{meV}$. This seems to us a reasonable value that is close to the well-known exciton binding energies in carbon nanotubes $[26,27]$. Note that this value may be scaled up or down by tuning the effective dielectric constant. We believe that the value used in this calculation $\varepsilon_{1}=6$ is reasonable for a semiconductor material having a band gap of about $2.5 \mathrm{eV}$. Solving Eq. (8) for spatially indirect excitons we obtain the variational parameter as $a=0.297 \mathrm{~nm}^{-1}$ and the exciton binding energy as $E_{X}=244,8 \mathrm{meV}$. Here we accounted for the spatial distribution of the indirect exciton wave function that spans over two parallel chains by using the reduced effective dielectric constant of $\varepsilon_{2}=4$. This value is obtained by weighted averaging of the effective dielectric constant of a single chain and the dielectric constant of vacuum surrounding the chains, $\varepsilon=1$. For the positively charged trion $X^{+}$, we obtain $a=0.297 \mathrm{~nm}^{-1}, c=0.298 \mathrm{~nm}^{-1}$, and the binding energy $E_{X^{+}}=274.7 \mathrm{meV}$. For the negatively charged trion $X^{-}$, we obtain $a=0.297 \mathrm{~nm}^{-1}, c=0.095 \mathrm{~nm}^{-1}$, and the binding energy $E_{X^{-}}=305.2 \mathrm{meV}$.

Let us now compare the calculation results obtained above with the experimental measurements of the excitonic fine structure in polyyne chains presented in Fig. 1(c). Using the parameters listed above we find the positive trion $X^{+}$peak shifted from the neutral exciton position by $15.9 \mathrm{meV}$ and the negative trion $X^{-}$resonance shifted from the neighboring $X^{+}$ peak by $30.5 \mathrm{meV}$, as Fig. 2 shows schematically. This appears to be in a very good agreement with the experimental data shown in Fig. 1(c).

One can easily understand why the negatively charged trion binding energy exceeds one of the positively charged trion looking at the trion relative motion wave functions shown in Figs. 3(d) and 3(e). The localization radius of a hole in a plane normal to the chain is smaller than the electron localization radius. This is why hole-hole repulsion in a positive trion is stronger than the electron-electron repulsion in a negative trion. This is why the negative trion appears to more strongly localized than the positive one. As a consequence, the binding energy of a negative trion is larger than one of the positive trion, so that the $X^{-}$peak is shifted to the lower energy than the $X^{+}$peak. Note that this order of trion states is different from one in GaAs-based quantum wires addressed in Ref. [23].

Next, from the model calculation, we obtain the energy splitting between spatially direct and indirect exciton states

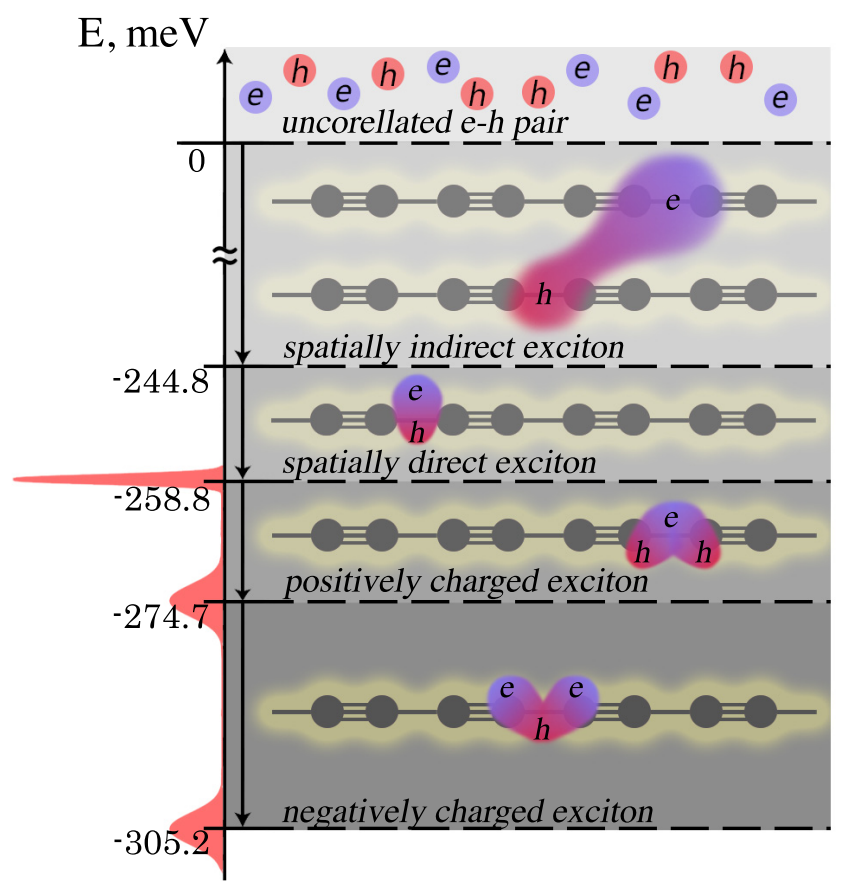

FIG. 2. The schematic energy diagram explaining the triple PL structure of in polyyne chains. Photoexcited electrons and holes may form spatially indirect excitons that do not emit light, as well as charged and neutral spatially direct excitons. The calculated transition energies of direct and indirect excitons as well as $X^{+}$and $X^{-}$ trions are indicated with respect to the transition energy between uncorrelated electron and hole states.

as $14 \mathrm{meV}$. This value that plays a role of the thermal hopping energy between the chains is governed by the difference in the shape of the Coulomb potential for two excitons as Fig. 3(c) shows. The calculated hopping energy is in a good agreement with the experimental data [9]. Indeed, the thermal dissociation of direct excitons leads to the broadening of exciton peaks that is apparent already at the temperatures of 60-90 K [Fig. 1(b)]. At room temperature, the time-resolved PL spectra of Ref. [9] show the presence of a fast nonradiative decay channel that is most likely associated with thermal hopping of carriers between chains. This channel is fully frozen out at the liquid helium temperature. From this ensemble of data, one can estimate the thermal hopping energy as $10-15 \mathrm{meV}$.

\section{CONCLUSION}

The developed variational model allowed us to confirm the origin of characteristic energy splittings experimentally observed in low-temperature PL spectra of polyyne chains. We predict the exciton and trion binding energies to be of the order of 250-300 meV. The triple PL structure that has repeatedly been observed in polyyne chains of different lengths is likely to be due to the spatially direct neutral exciton, positive and negative trion peaks, respectively. The nonradiative exciton decay channel observed in the time-resolved PL spectra at room temperature is most probably associated with the thermal hopping of one of the carriers forming the exciton between parallel polyyne chains. Excitons are not destroyed by this hopping process but they become radiatively inactive 


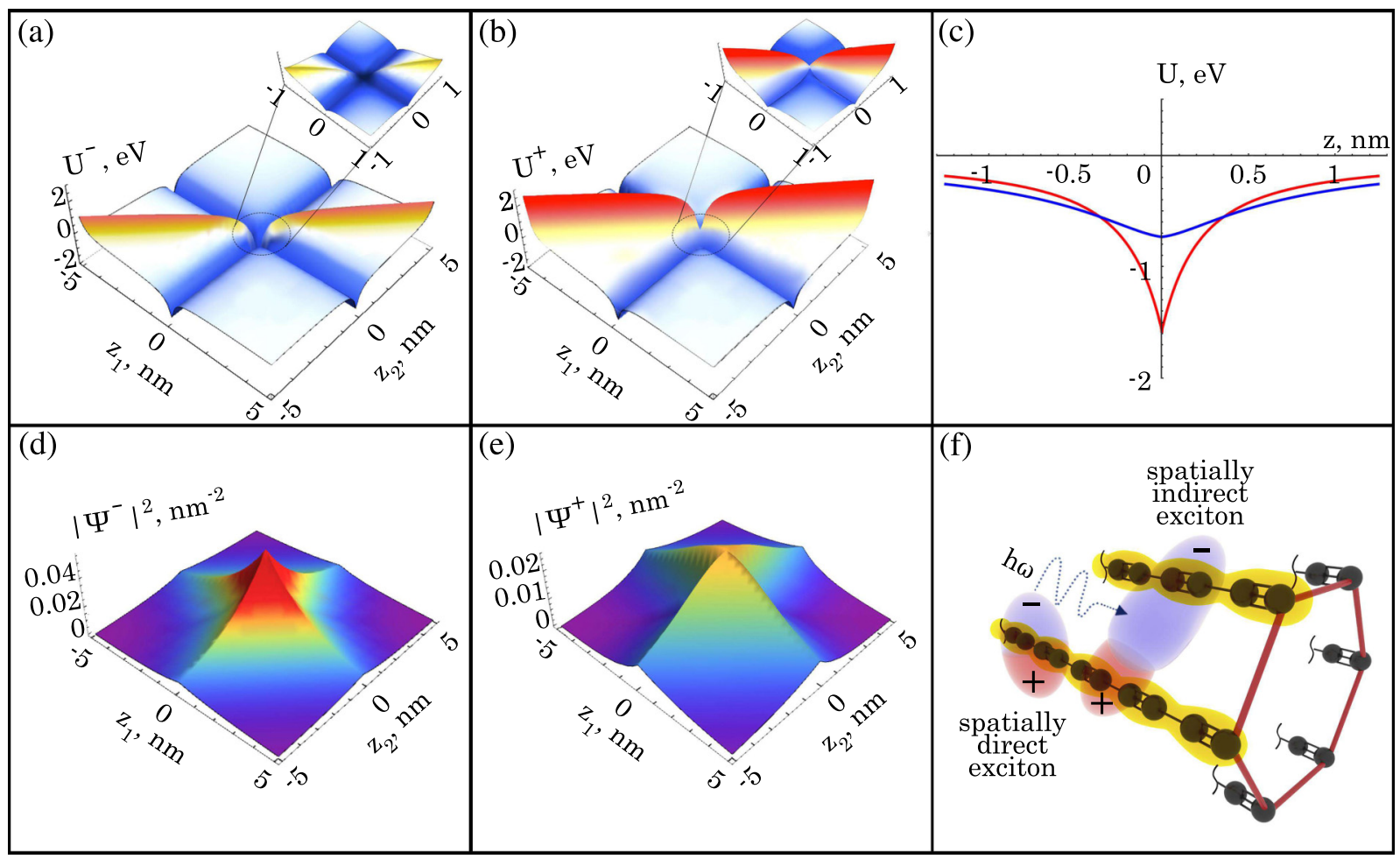

FIG. 3. The charged exciton structure: Panels (a) and (b) show the potentials of Coulomb interaction for the negatively charged trion $U^{-}\left(z_{1}, z_{2}\right)$ and positively charged trion $U^{+}\left(z_{1}, z_{2}\right)$, respectively. Panel (c) shows the calculated interaction potentials corresponding to the exciton states: The red curve shows the Coulomb potential energy of a spatially direct exciton and the blue one shows the Coulomb energy of a spatially indirect exciton. Panels (d) and (e) show negatively charged trion wave function $\Psi^{-}\left(z_{1}, z_{2}\right)$ and positively charged trion wave function $\Psi^{+}\left(z_{1}, z_{2}\right)$, respectively. Panel (f) shows the schematic illustration of spatially direct and indirect excitons that may be formed in polyyne chains bundled in a hexagonal Van der Waals quasicrystal.

or dark. We realize that the proposed model has several shortcomings. First, we assumed the carbon chains to be infinitely long. We opted for keeping the model as simple as possible having in mind that, experimentally, the exciton-trion triplet appears to be essentially independent of the length of the chain. The effect of the exciton center-of-mass confinement on the length of the chain is addressed in the Supplemental material. We find that if the distance between gold nanoparticles confining the carbin chain is $>50 \mathrm{~nm}$, the finite-length effect on the exciton energy is negligible. Another limitation of the validity of our approach comes from the limited accuracy of the effective mass approximation in nanosystems of such a small size as bundles of polyyne chains composed by 10-20 atoms each. Still, we are confident that the method predicted a correct order of magnitude for the exciton and trion binding and hopping energies, as the comparison with available experimental data certifies. Further experimental and theoretical studies are needed to reveal the spin structure and transport properties of quasiparticles in linear carbon chains.

\section{ACKNOWLEDGMENTS}

The work is supported by Westlake University, Project No. 041020100118, and the Program 2018R01002 funded by Leading Innovative and Entrepreneur Team Introduction Program of Zhejiang and by MSHE within the State assignment VISU 0635-2020-0013. A.K. acknowledges the support from Rosatom within the Road map for quantum computing. The S.D.'s work is supported from the Grant of the President of the Russian Federation for state support of young Russian scientists No. MK-5318.2021.1.2. S.B., A.Z., and P.L. acknowledge RFBR Grants No. 20-52-12026 and No. 20-0200919. A.Z. acknowledge financial support by the Russian Science Foundation (RScF) Grant No. 20-72-10145.
[1] G. Casillas, A. Mayoral, M. Liu, A. Ponce, V. I. Artyukhov, B. I. Yakobson, and M. Jose-Yacaman, New insights into the properties and interactions of carbon chains as revealed by HRTEM and DFT analysis, Carbon 66, 436 (2014).
[2] M. Liu, V. I. Artyukhov, H. Lee, F. Xu, and B. I. Yakobson, Carbyne from first principles: Chain of $\mathrm{C}$ atoms, a nanorod or a nanorope, ACS Nano 7, 10075 (2013). 
[3] C. S. Casari, M. Tommasini, R. Tykwinski, and A. Milani, Carbon-atom wires: 1-D systems with tunable properties, Nanoscale 8, 4414 (2016).

[4] V. I. Artyukhov, M. Liu, and B. I. Yakobson, Mechanically induced metalâinsulator transition in carbyne, Nano Lett. 14, 4224 (2014).

[5] O. Cretu, A. R. Botello-Mendez, I. Janowska, C. Pham-Huu, J.-C. Charlier, and F. Banhart, Electrical transport measured in atomic carbon chains, Nano Lett. 13, 3487 (2013).

[6] A. La Torre, A. Botello-Mendez, W. Baaziz, J.-C. Charlier, and F. Banhart, Strain-induced metalâsemiconductor transition observed in atomic carbon chains, Nat. Commun. 6, 6636 (2015).

[7] L. Shi, P. Rohringer, K. Suenaga, Y. Niimi, J. Kotakoski, J. C. Meyer, H. Peterlik, M. Wanko, S. Cahangirov, A. Rubio, Z. Lapin, L. Novotny, P. Ayala, T. Pichler, Confined linear carbon chains as a route to bulk carbyne, Nat. Mater. 15, 634 (2016).

[8] S. Kutrovskaya, I. Chestnov, A. Osipov, V. Samyshkin, I. Sapegina, A. Kavokin, and A. Kucherik, Electric field assisted alignment of monoatomic carbon chains, Sci. Rep. 10, 9709 (2020).

[9] S. Kutrovskaya, A. Osipov, S. Baryshev, A. Zasedatelev, V. Samyshkin, S. Demirchyan, O. Pulci, D. Grassano, L. Gontrani, R. R. Hartmann, M. E. Portnoi, A. Kucherik, P. Lagoudakis, and A. Kavokin, Excitonic fine structure in emission of linear carbon chains, Nano Lett. 20, 6502 (2020).

[10] E. F. Gross and N. A. Karryjew, The optical spectrum of the exciton, Dokl. Akad. Nauk SSSR 84, 471 (1952).

[11] A. I. Ekimov, Al. L. Efros, and A. A. Onushchenko, Quantum size effect in semiconductor microcrystals, Solid State Commun. 56, 921 (1985).

[12] M. A. Boles, D. Ling, T. Hyeon, and D. V. Talapin, The surface science of nanocrystals, Nat. Mater. 15, 141 (2016).

[13] A. L. Efros, M. Rosen, M. Kuno, M. Nirmal, D. J. Norris, and M. Bawendi, Band-edge exciton in quantum dots of semiconductors with a degenerate valence band: Dark and bright exciton states, Phys. Rev. B 54, 4843 (1996).

[14] M. V. Kovalenko, L. Manna, A. Cabot, Z. Hens, D. V. Talapin, C. R. Kagan, V. I. Klimov, A. L. Rogach, P. Reiss, D. J. Milliron, P. Guyot-Sionnnest, G. Konstantatos, W. J. Parak, T. Hyeon, B. A. Korgel, C. B. Murray, and W. Heiss, Prospects of nanoscience with nanocrystals, ACS Nano 9, 1012 (2015).
[15] F. Liu, L. Biadala, A. V. Rodina, D. R. Yakovlev, D. Dunker, C. Javaux, J.-P. Hermier, A. L. Efros, B. Dubertret, and M. Bayer, Spin dynamics of negatively charged excitons in $\mathrm{CdSe} / \mathrm{CdS}$ colloidal nanocrystals, Phys. Rev. B 88, 035302 (2013).

[16] E. V. Shornikova, D. R. Yakovlev, L. Biadala, S. A. Crooker, V. V. Belykh, M. V. Kochiev, A. Kuntzmann, M. Nasilowski, B. Dubertret, and M. Bayer, Negatively charged excitons in CdSe nanoplatelets, Nano Lett. 20, 1370 (2020).

[17] S. Gupta, A. Kutana, B. I. Yakobson, Heterobilayers of 2D materials as a platform for excitonic superfluidity, Nat. Commun. 11, 2989 (2020).

[18] A. V. Kavokin, J. J. Baumberg, G. Malpuech, and F. P. Laussy, Microcavities (Oxford University, New York, 2007).

[19] M. Semina, R. Sergeev, and R. Suris, Binding energies of 2D laterally confined trions, Phys. E (Amsterdam) 40, 1357 (2008).

[20] D. K. Efimkin and A. H. MacDonald, Many-body theory of trion absorption features in two-dimensional semiconductors, Phys. Rev. B 95, 035417 (2017).

[21] A. V. Kavokin, A. I. Nesvizhskii, and R. P. Seisyan, Exciton in a semiconductor quantum well subjected to a strong magnetic field, Semiconductors 27, 530 (1993).

[22] E. Courtade, M. Semina, M. Manca, M. M. Glazov, C. Robert, F. Cadiz, G. Wang, T. Taniguchi, K. Watanabe, M. Pierre, W. Escoffier, E. L. Ivchenko, P. Renucci, X. Marie, T. Amand, and B. Urbaszek, Charged excitons in monolayer WSe 2: Experiment and theory, Phys. Rev. B 96, 085302 (2017).

[23] M. Semina, R. Sergeev, and R. Suris, The binding energy of excitons and $\mathrm{X}+$ and $\mathrm{X}$ - trions in one-dimensional systems, Semiconductors 42, 1427 (2008).

[24] R. Loudon, One-dimensional hydrogen atom, Am. J. Phys. 27, 649 (1959).

[25] See Supplemental Material at http://link.aps.org/supplemental/ 10.1103/PhysRevResearch.3.013071 for the detailed formalism for the three variational parameters model, where also the finitelength effect is discussed.

[26] A. R. Amori, Z. Hou, and T. D. Krauss, Excitons in singlewalled carbon nanotubes and their dynamics, Annu. Rev. Phys. Chem. 69, 81 (2018).

[27] E. Mostaani, B. Monserrat, N. D. Drummond, and C. J. Lambert, Quasiparticle and excitonic gaps of one-dimensional carbon chains, Phys. Chem. Chem. Phys. 18, 14810 (2016). 